Provided for non-commercial research and education use. Not for reproduction, distribution or commercial use.

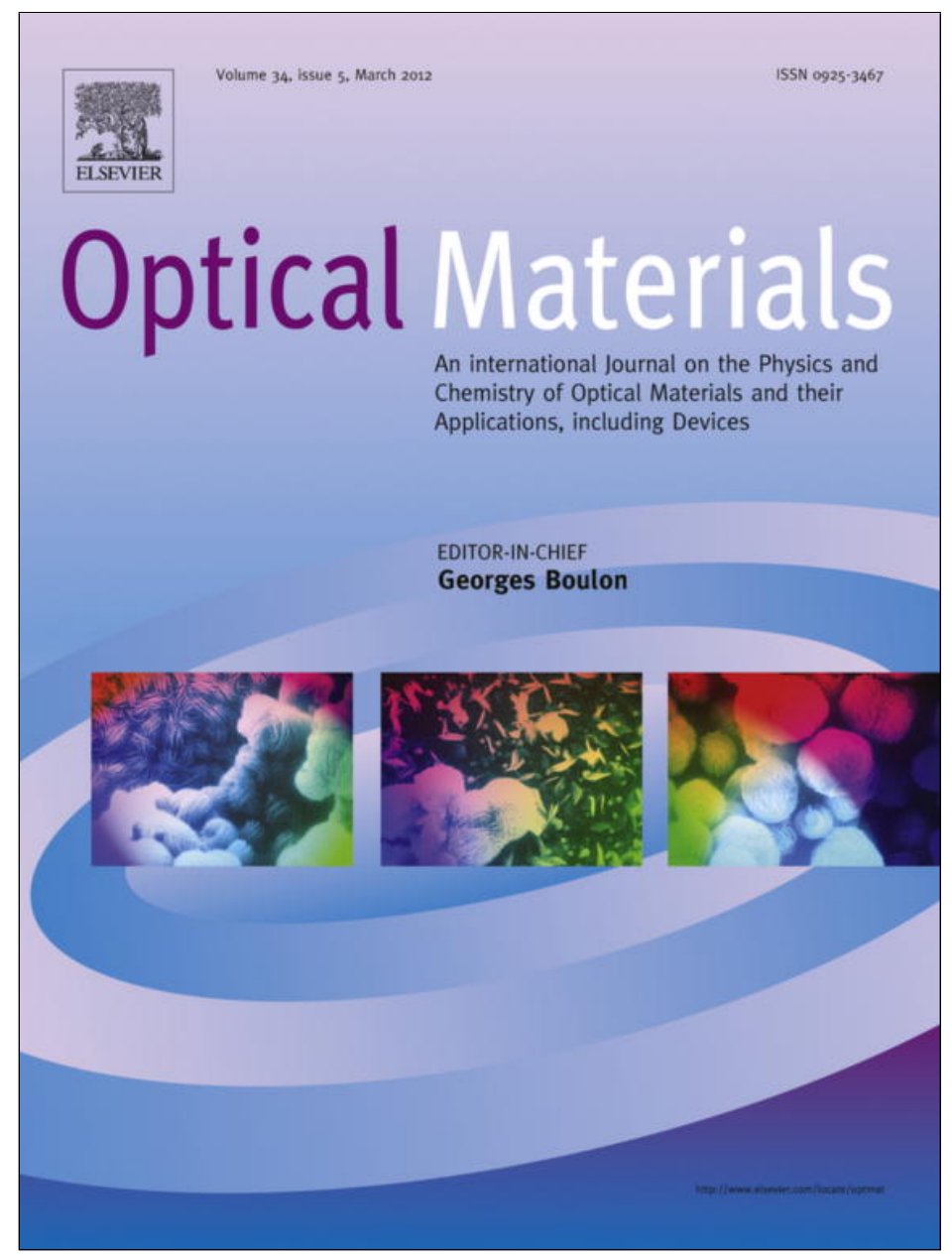

This article appeared in a journal published by Elsevier. The attached copy is furnished to the author for internal non-commercial research and education use, including for instruction at the authors institution and sharing with colleagues.

Other uses, including reproduction and distribution, or selling or licensing copies, or posting to personal, institutional or third party websites are prohibited.

In most cases authors are permitted to post their version of the article (e.g. in Word or Tex form) to their personal website or institutional repository. Authors requiring further information regarding Elsevier's archiving and manuscript policies are encouraged to visit:

http://www.elsevier.com/copyright 


\title{
An intrinsic luminescence in binary lead silicate glasses
}

\author{
A.F. Zatsepin ${ }^{\text {a }}$, I.S. Zhidkov ${ }^{\text {a }}$, A.I. Kukharenko ${ }^{\text {a }}$, D.A. Zatsepin ${ }^{\text {a,b,* }}$, M.P. Andronov ${ }^{\text {a }}$, S.O. Cholakh ${ }^{\text {a }}$ \\ ${ }^{a}$ Ural Federal University, Mira Str. 19, 620002 Yekaterinburg, Russia \\ ' Institute of Metal Physics, RAS Ural Div., Kovalevskoi Str. 18, 620990 Yekaterinburg, Russia
}

\section{A R T I C L E I N F O}

\section{Article history:}

Received 19 July 2011

Received in revised form 7 November 2011

Accepted 9 November 2011

Available online 20 December 2011

\section{Keywords:}

Lead silicate glass

Localized states

Photoluminescence

Temperature quenching

Street's law

Mott's law

\begin{abstract}
A B S T R A C T
The low-temperature photoluminescence (PL) of $x \mathrm{PbO} \cdot(1-x) \mathrm{SiO}_{2}$ glasses $(x=0.20-0.75)$ was studied at $T=10 \mathrm{~K}$. The recorded PL-spectra are a superposition of three spectral components with maxima located at $1.8 \mathrm{eV}$ (identified as $\mathrm{Pb} 6 p \rightarrow$ metal-bridging $02 p$ radiative electron transition, the " $R$ "-band), $2.0 \mathrm{eV}$ ( $\mathrm{Pb} 6 p \rightarrow$ non-bridging $02 p$, the "O"-band) and $2.55 \mathrm{eV}(\mathrm{Pb} 6 p \rightarrow \mathrm{Pb} 6 s$, the " $\mathrm{B}$ "-band), respectively. It was found the essential link for " $R$ ", " $O$ " and " $B$ " PL-bands with chemical composition $x$ of the glasses under study. These concentration dependences are expressed as mutual PL-intensity variations for each recorded luminescence band that allowed to determine their origin. The shape of established dependences well coincides with numerical data on NBO- and MBO-density of chemical bonding, reported previously.

The overall PL-manner within the temperature range of $10-295 \mathrm{~K}$ is described by an empirical Street's law. It was shown that experimental photoluminescence quenching curves may be precisely approximated as a superposition of Mott relationships for nonequivalent luminescence centers. The obtained distribution of PL-centers on the activation energy for luminescence quenching reflects the essential donation of the low-energy states into the overall PL-process. The width of this energy distribution affects by the type of PL-emission band and the disordering degree in the arrangement of local PL-centers of a certain kind.
\end{abstract}

() 2011 Elsevier B.V. All rights reserved.

\section{Introduction}

Lead silicate glasses are widely used in different commercial applications such as optical lenses, electronic and optoelectronic devices, etc. due to their high refractive index, large secondary electron emission coefficient, overall chemical stability and low melting point [1-3]. The successful application of lead-silicate glasses is determined by specific peculiarities of their electron energy spectrum which is characterized by the high density of localized electronic states in the tails of the energy bands (the so-called "tail-states").

Well known $[4,5]$ that the long-wavelength limit of the chargecarrier mobility is much higher than the fundamental absorption edge in lead silicate glasses. Therefore, the origin of luminescentoptical properties will be essentially determined by electron transitions between the localized tail-states of the energy band spectrum [6]. At the same time, it is impossible to improve the functionality of a material, based on lead-silicate glasses, without carrying out a detailed study of the nature of low-energy electronic excitations, the regularities of intrinsic luminescence and general

\footnotetext{
* Corresponding author at: Ural Federal University, Mira Str. 19, 620002 Yekaterinburg, Russia. Tel.: +7 3433759788.

E-mail addresses: nexcom@list.ru, zats@dpt.ustu.ru (D.A. Zatsepin).
}

relationship between the chemical composition, atomic structure and physical property.

The $x \mathrm{PbO} \cdot(1-x) \mathrm{SiO}_{2}$ system has a wide range of glass formation, up to $x=0.90$ area. A distinctive feature of lead-silicate glasses is the existence of two concentration areas which are significantly dissimilar from each other by the atomic short-range ordering and, hence, electronic structure [7-11]. The low-lead glassy matrix (when $x<0.50$ ) is mainly composed of interlinked $\mathrm{SiO}_{4}$-tetrahedra because the $\mathrm{Pb}$-ions modify the silica-oxygen net by forming the bonds of $\mathrm{Si}-\mathrm{O}-\mathrm{Pb}$ type [8]. As for the atomic short-range ordering in the inverted glasses (i.e. $x>0.50$ ), it is characterized by the presence of $\mathrm{PbO}_{n}$-polyhedra, which are able to form an independent amorphous structure within the shell-material. These structural glass-forming units appear due to the "bridge" chemical bonding of the $\mathrm{Pb}-\mathrm{O}-\mathrm{Pb}$ type. A wide variety in bonding type between oxygen atoms, lead atoms and silica ones result in the formation of diverse structural motifs that are determining the majority of electron-optical properties of the glass $[9,10]$. Unfortunately, there is a lack of detailed discussions on the possible correlation between the glass-forming structural unit and overall fluorescent characteristics of $\mathrm{PbO} \mathrm{SiO}$ glassy system [6,12,13].

In general, the luminescent characteristics of lead silicate glasses have been studied using the samples with low-lead content, i.e. within $x \leqslant 0.40$ areas. The origin of luminescence centers was presumably attributed to the radiative relaxation of 
localized electronic states or intra-center transitions in $\mathrm{Pb}^{2+}$-ion $[6,12,14,15]$. Inter alia, the authors of Ref. [13] supposed the existence of several luminescent centers (LC) that are of the same origin but with different Stokes shift. In the frame of their model approach, the luminescence characteristics of these LC's are essentially affected by the lead ions local environment. In addition, the growth and increasing of PbO-phase in the glass, will lead, on the whole, to a significant concentration quenching of a luminescence. The intensity of the glow begins to weaken noticeably even at liquid nitrogen temperatures [12]. At room-temperatures the luminescence is almost quenched for high-lead silicate glasses, and for the samples with $x \leqslant 0.45$ one can record only a slight glow. Finally, the analysis of previously reported data indicates the complex nature of intrinsic luminescence in lead-silicate glasses and, hence, the ambiguity of its interpretation.

In the current manuscript we present the study of intrinsic luminescence in $\mathrm{PbO}-\mathrm{SiO}_{2}$ glassy system within the temperature range of 10-295 K taking into account the short-range order inversion. Also the variations in the nature of localized electronic states and the matrix disordering will be discussed.

\section{Sample preparation and experimental details}

Binary glasses of $x \mathrm{PbO} \cdot(1-x) \mathrm{SiO}_{2}$ composition $(x=0.20-0.75)$ were synthesized in the temperature range of $T=1300-1450{ }^{\circ} \mathrm{C}$ from high-purity initial components that were taken in strictly appropriate ratio (Ural Federal University, Yekaterinburg, Russia). The performed chemical analysis of binary glasses under study displays the composition which corresponds to the initial synthesis composition up to $1 \mathrm{~mol}$.\%.

Room temperature $(T=295 \mathrm{~K})$ PL-spectra in the wavelength range $1.5-5.0 \mathrm{eV}$ were recorded by using a spectrometer operating in photon-counting mode. The PL has been selectively excited in the wavelength range $3.0-5.5 \mathrm{eV}$ by using a $400 \mathrm{~W}$ deuterium lamp. The experimental spectra of luminescence excitation were normalized to an equal number of incident photons.

The low-temperature $(T=10 \mathrm{~K})$ PL-spectra and PL-excitation spectra, as well as the PL temperature dependences, were measured at SUPERLUMI end-station (HASYLAB-DESY, Hamburg, Germany) using a high-brilliance synchrotron radiation. PL excitation spectra were measured in the $4-12 \mathrm{eV}$ range and normalized to an equal number of incident photons. The luminescence spectra were recorded with the help of $0.3 \mathrm{~m}$ ARC Spectra Pro-308i monochromator and the Hamamatsu R6358P photomultiplier.

\section{Results}

Fig. 1 shows the low-temperature PL-spectra of binary lead silicate glasses that were measured at photon excitation energy $E_{e x}$ $c=3.9 \mathrm{eV}$. It is possible to decompose these spectra into three components with the maxima located at $1.8 \mathrm{eV}$ (named as the " $R$ "-band), $2.0 \mathrm{eV}$ (the " $O$ "-band) and $2.55 \mathrm{eV}$ (the " $B$ "-band), respectively. Full width at half maximum (FWHM) for the $R-, O-$ and $B$-bands remains invariable and is in the range of $0.4-0.5 \mathrm{eV}$. When the composition factor $x$ takes the value $x=0.50-0.55$, the $R$-, $O$ - and $B$-bands display approximately the same intensity within the measurement error. The PL-spectra of high-lead samples $(x \geqslant 0.65)$ are characterized by the majority of $R$-band with simultaneous intensity diving for the rest bands.

Concentration dependence of the integrated PL-intensities for $R-B$ bands are shown in Fig. 2. The intensity of the high-energy $B$-band decreases regularly with increasing $\mathrm{PbO}$-concentration while the intensity of the $R$-band, on the contrary, rises up. As for the contribution of the $O$-band PL-intensity, it is increasing with PbO-concentration, becomes maximum in the sample with

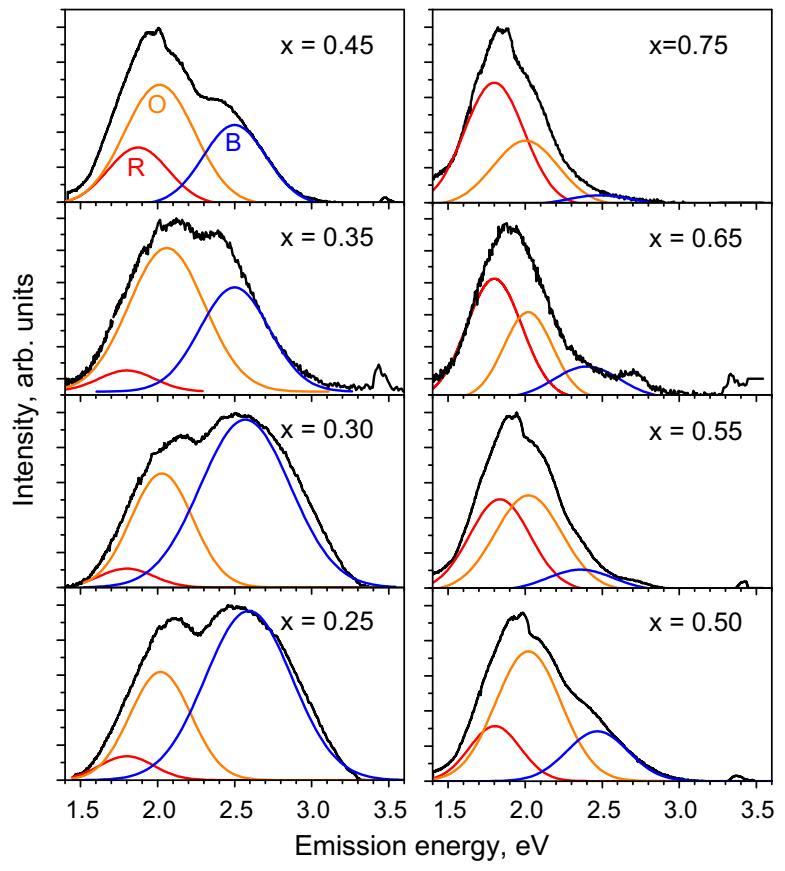

Fig. 1. Photoluminescence spectra of $x \mathrm{PbO} \cdot(1-x) \mathrm{SiO}_{2}$ glasses. $E_{e x c}=3.9 \mathrm{eV}, T=$ $10 \mathrm{~K}$.

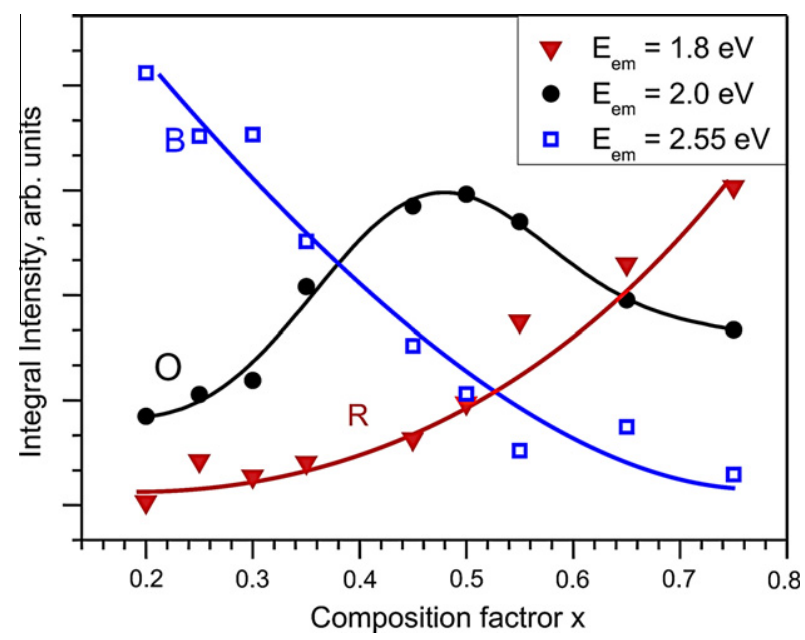

Fig. 2. The intensity of $R, O$ and $B$-bands of the PL-spectrum versus the composition factor $x$.

$x=0.50$, and then, falls down again. Also, there is nearly homogeneous decreasing of the overall PL-spectrum intensity when the temperature rises up, and dynamics of the PL temperature quenching strongly correlates with the composition of the samples under study. Fig. 3 displays the PL-spectra of glasses with $x=0.25$ and $x=0.50$ at $10 \mathrm{~K}, 67 \mathrm{~K}$ and $125 \mathrm{~K}$. One can see from this figure that with increasing of lead oxide concentration in the glass, the luminescence is quenched at lower temperatures. For this reason, we have observed the PL at room temperatures only in low-lead glasses (see Fig. 4).

We have separated three bands with maxima located at $2.05 \mathrm{eV}$, $2.35 \mathrm{eV}$ and $2.75 \mathrm{eV}$ using a decomposition of the spectrum into Gaussian components. The band widths of PL-spectra recorded at $T=295 \mathrm{~K}$ are characterized with the same values as at $T=10 \mathrm{~K}$ (the FWHM $=0.4-0.5 \mathrm{eV}$ ). When $x=0.25-0.30$ the majority of PL-spectrum is determined by the band with a maximum at $2.35 \mathrm{eV}$, and when $x=0.45$ - the band at $2.05 \mathrm{eV}$ becomes 


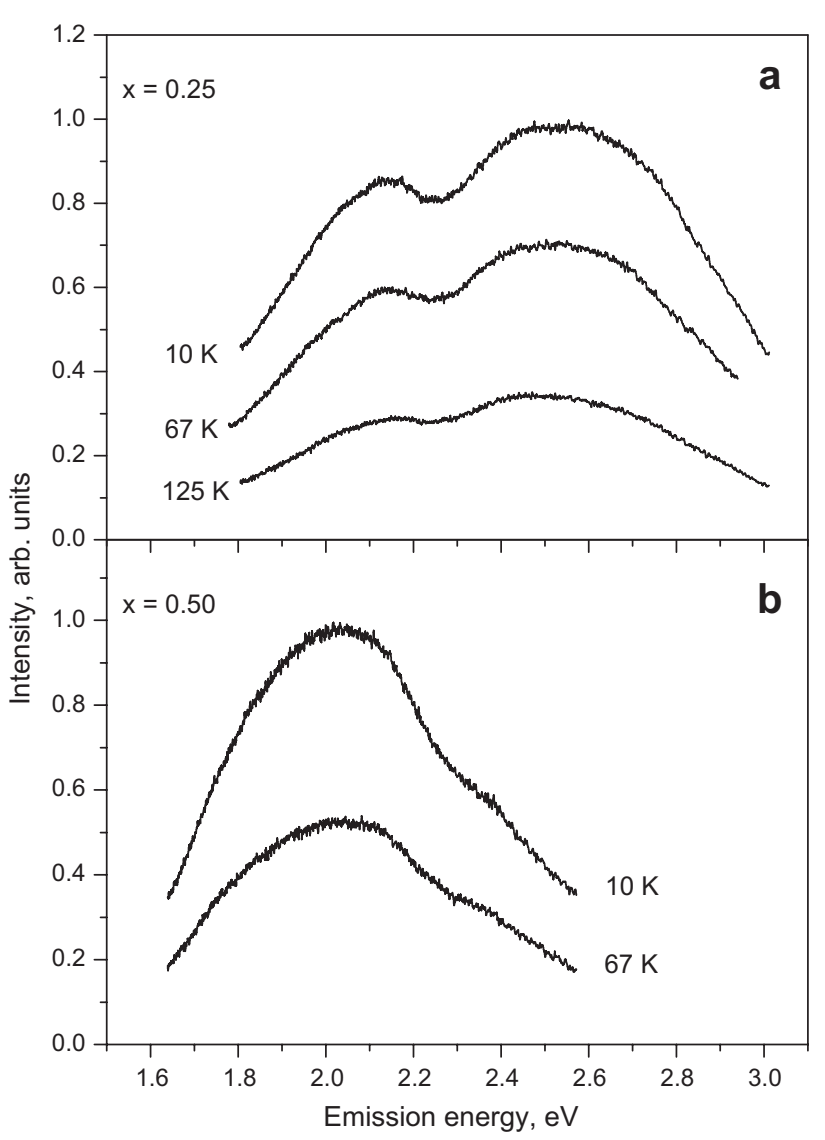

Fig. 3. Temperature evolution of photoluminescence spectra for glasses with different composition factor (a) $x=0.25$; (b) $x=0.50$. $E_{\text {exc }}=4.0 \mathrm{eV}$.

dominating. The $2.75 \mathrm{eV}$ band has nearly the average mean of intensity in the sample with $x=0.25$ and decays with increasing of PbO-content in the sample. The character of intensity variations for $2.05 \mathrm{eV}, 2.35 \mathrm{eV}$ and $2.75 \mathrm{eV}$ bands with increasing of PbO-content allow to link them with the $R$-, $O$ - and $B$-bands in the low-temperature PL-spectra, respectively (see Fig. 1).

PL-excitation spectra at $T=10 \mathrm{~K}$ are shown in Fig. 5. For lowlead samples the luminescence excitation spectra were obtained for the energy value of $E_{\mathrm{em}}=2.5 \mathrm{eV}$, and in the case of high-lead samples - for $E_{\mathrm{em}}=2.0 \mathrm{eV}$. The spectra of all samples under study have a maximum at $3.8 \mathrm{eV}$, and the energy location of this maximum depends on the PbO-concentration. When the composite factor $x$ changes in the direction of increasing, the excitation maximum starts to shift to the low-energy area, that is, probably, linked with the transformation of the $E_{\mathrm{g}}$ band gap. Described above data well coincides with that reported in Ref. [10,11]. There it was shown and proved that increasing $\mathrm{PbO}$-content in the glass results in the decreasing of band gap $E_{\mathrm{g}}$, namely from $3.74 \mathrm{eV}(x=0.20)$ up to $2.8 \mathrm{eV}$ (for $x=0.75$ ). Thus, one can assume that the observed PL of the samples under study is excited by means of electron transitions between localized tails-states.

Luminescent activity of glasses usually decreases with increasing temperature, while the dramatic PL quenching already starts at $T=10 \mathrm{~K}$. Fig. 6 displays the curves of the PL- quenching of the samples under study with $x=0.20 ; 0.25 ; 0.50$ and $0.55\left(E_{\text {exc }}=4.0 \mathrm{eV}\right)$. These curves were measured at the luminescence maxima of the samples, i.e. at $2.5 \mathrm{eV}$ for low-lead samples and at $2.1 \mathrm{eV}$ for high-lead glasses.

The obtained dependences $I_{L}(T)$ are well described by an empirical Street law [16, 17]:

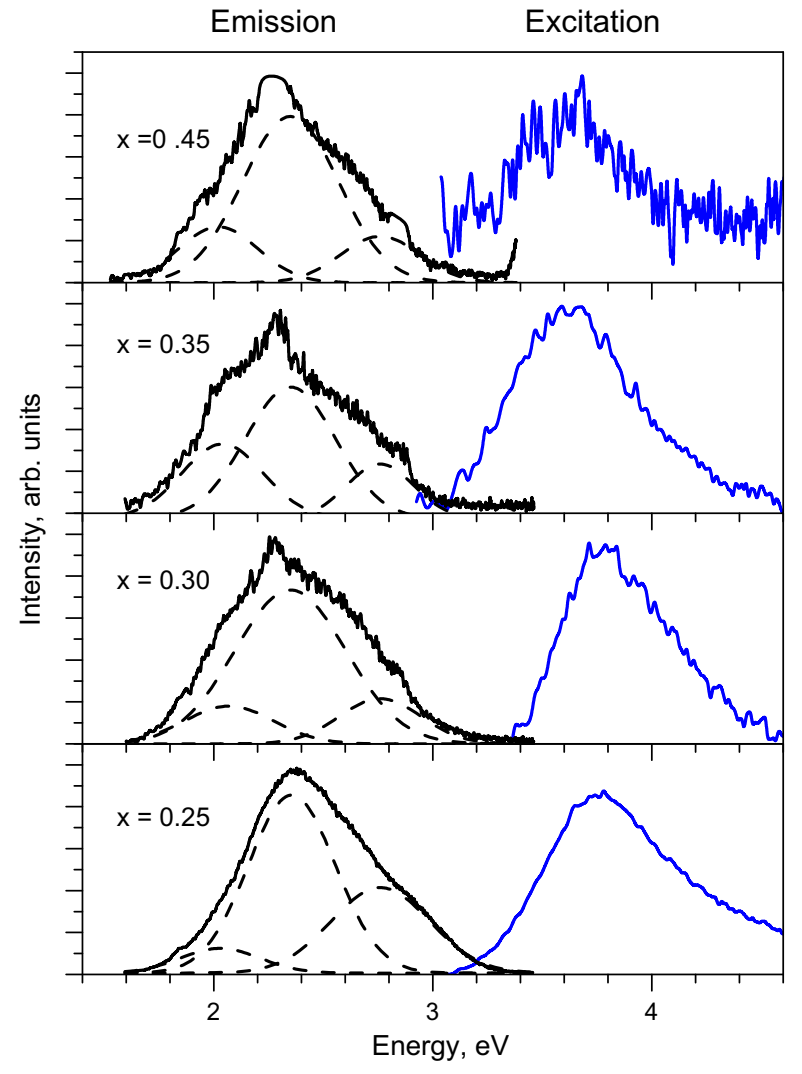

Fig. 4. PL-spectra $\left(E_{\text {exc }}=4.0 \mathrm{eV}\right)$ and excitation PL-spectra $\left(E_{\text {em }}=2.3 \mathrm{eV}\right)$ of glasses at a room temperature.

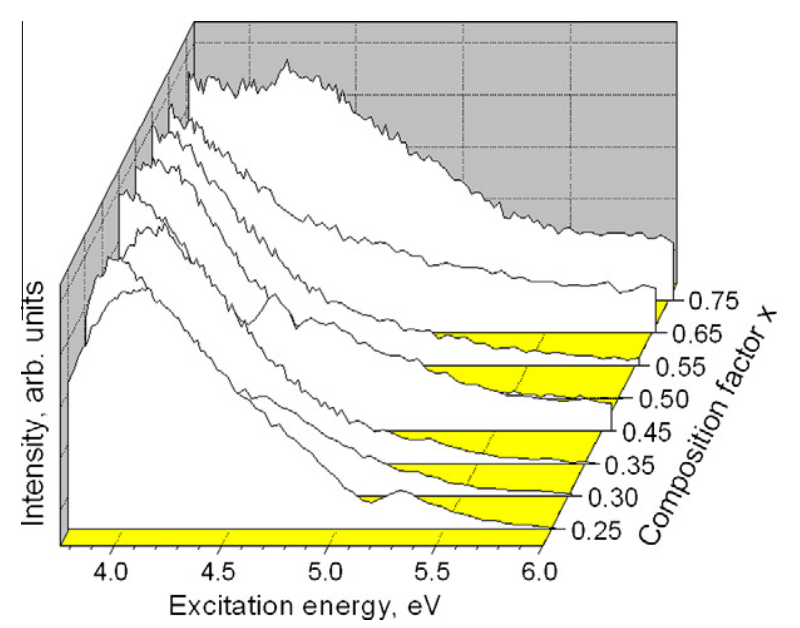

Fig. 5. Excitation spectra $\left(E_{e m}=2.5 \mathrm{eV}\right)$ of $x \mathrm{PbO} \cdot(1-x) \mathrm{SiO}_{2}$ glasses versus composition factor $x=0.25-0.75$ at $T=10 \mathrm{~K}$.

$I_{L}(T)=I_{0}^{S}\left[1+p_{s} \exp \left(\frac{T}{T_{0}}\right)\right]^{-1}$,

where $p_{s}$ is characteristic parameter and $I_{0}^{S}$ - an intensity at $T=10 \mathrm{~K}$. The $T_{0}$ factor of Street function reflects the presence of dispersion in structural and energy parameters of emission centers, and, in particular, is linked with the length of the band-tails of localized states [16]. The results of experimental curves processing with the help of Eq. (1) are presented in Table. 1. It is obvious that with increasing of lead oxide content, a decreasing of $T_{0}$ factor takes place that is reflecting the change in the slope of temperature quenching curves. 


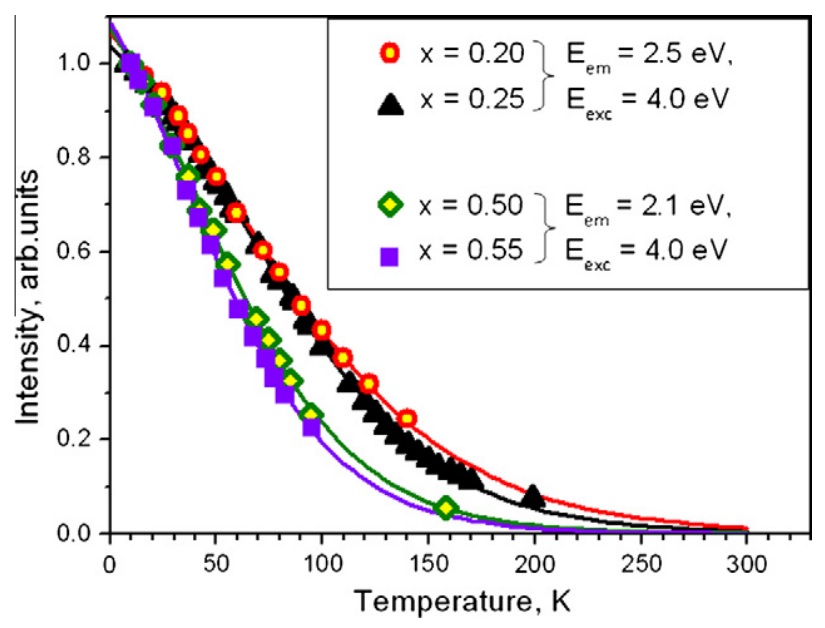

Fig. 6. Temperature quenching of photoluminescence. Lines - an approximation using Eq. (2); symbols - an experimental data.

Table 1

Fitting parameters for the experimental curves approximated by Street law (Eq. (1)).

\begin{tabular}{llll}
\hline$x$ & $T_{0}(\mathrm{~K})$ & $I_{0}^{S}$ (arb. units) & $p_{S}$ \\
\hline 0.20 & 42.549 & 22.609 & 0.203 \\
0.25 & 51.357 & 45.582 & 0.322 \\
0.50 & 36.096 & 19.453 & 0.312 \\
0.55 & 33.754 & 12.336 & 0.333 \\
\hline
\end{tabular}

In general, the luminescence of glasses can be regarded as a set of intra-center radiative transitions of similar physical nature in some statistical ensemble of local luminescence centers which are differing in energy. Since the quenching centers with the same activation energy are usually the subject to the ratio of Mott, so to analyze the origin and mechanisms of luminescence suppression is advisable to use an approach proposed in Refs. $[18,19]$. Under this approach, the expression for $I_{L}(T)$ can be represented as:

$I_{L}(T)=I_{0}^{M} \int_{0}^{\infty}\left[1+p_{M} \exp \left(-\frac{E_{a}}{k T}\right)\right]^{-1} g\left(E_{a}\right) d E_{a}$,

where $I_{0}^{M}$ is an intensity at $T=10 \mathrm{~K}, p_{M}$ - characteristic parameter, $E_{a}$ - activation energy, $k$ - Boltzmann constant, and $g\left(E_{a}\right)$ - distribution function of emission centers depending on the activation energy [18]:

$g\left(E_{a}\right)=\frac{1}{w \sqrt{\pi / 2}} \exp \left(-\frac{2\left(E_{a}-E_{0}\right)^{2}}{w^{2}}\right)$

where $E_{0}$ is maximum of distribution and $w$ - distribution width.

The distribution of luminescence centers on the activation energy of quenching process is shown in Fig. 7. One can see that an increasing of lead concentration in the glass will force a decrease of distribution width $w$ and shifts the maximum distribution $E_{0}$ to the low-energy region. The values calculated with the help of Eqs. (2) and (3) are presented in Table. 2.

The obtained results show that the low-temperature PL spectra of binary lead silicate glasses revealed several bands, effectively excited in the interband transitions with the energy of $3.8 \mathrm{eV}$. The character of the dependences of the PL-intensity on the glass composition (Fig. 2), dissimilar efficiency of temperature quenching for the corresponding luminescence bands - all these reflect that several types of a nonequivalent luminescence centers participate in the radiative relaxation of the low-energy electron excitations in the $x \mathrm{PbO} \cdot(1-x) \mathrm{SiO}_{2}$ glassy system. In addition, the quantitative proportion between them essentially depends on the composition of the glass.

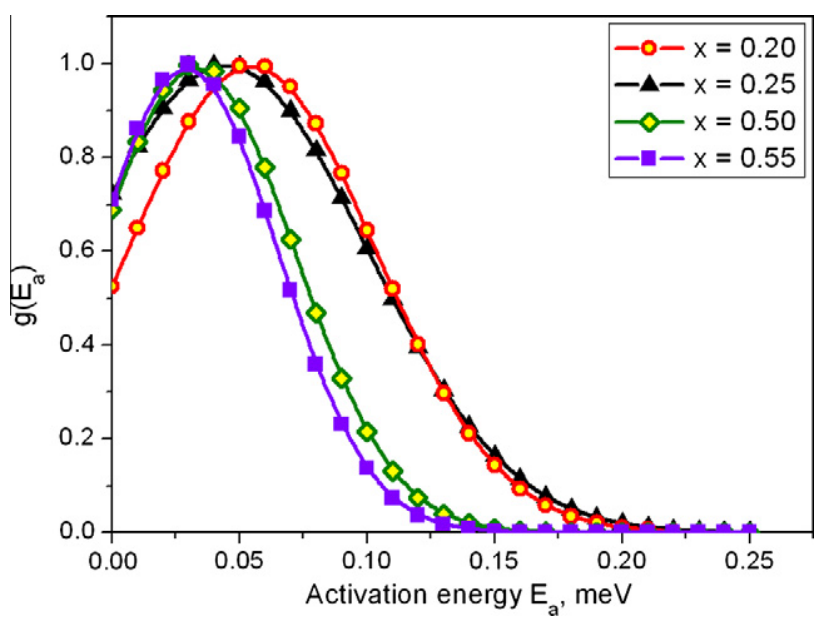

Fig. 7. Distribution of emission centers for activation energy of temperature quenching. $E_{e m}$ and $E_{e x c}$ are similar to those in Fig. 6 .

Table 2

Fitting parameters for the experimental quenching curves with Eqs. (2) and (3).

\begin{tabular}{lllll}
\hline$x$ & $p_{M}\left(\times 10^{3}\right)$ & $w(\mathrm{eV})$ & $E_{0}(\mathrm{eV})$ & $I_{0}^{M}$ (arb. units) \\
\hline 0.20 & 7.075 & 0.082 & 0.055 & 178.079 \\
0.25 & 3.872 & 0.094 & 0.045 & 313.783 \\
0.50 & 3.802 & 0.065 & 0.033 & 190.931 \\
0.55 & 3.553 & 0.060 & 0.029 & 129.878 \\
\hline
\end{tabular}

\section{Discussion}

The obtained results display that the PL-spectra of the glasses under study are the superposition of the individual $R, O$ and $B$ emission bands (see Fig. 1). Most clearly the specific of ternary glasses with different compositions can be seen at the temperatures of liquid helium, i.e. when the influence of luminescence temperature quenching is minimized. A different kind of concentration dependences for PL-intensities of $R, O$ and $B$-bands (Fig. 2), as well as their temperature dependences (Fig. 3 and 6), allow to conclude that these bands correspond to three distinguish types of centers which have different origin. Pertinent to note that the possible existence in the glass structure several type of PL-centers with different Stokes shift have been expressed previously in Ref. [13].

To clarify the nature of PL-centers described above, it is needed to consider the atomic structure of glasses in details. Please note, that in the composition range of $x=0.45-0.50$ the glasses under study are experiencing the strong atomic and energy structure transformations $[7,8,10,11]$. There is also evidence that the glass structure forming motives, are the subject to vary considerably. In particular, it was shown that in a wide glass-forming range there are three types of structural unit groups with different states of oxygen atom $[8,9]$. It is decided to allocate the bridging oxygen bonding $\mathrm{Si}-\mathrm{O}-\mathrm{Si}$ (usually denoted as $\mathrm{BO}$ ), non-bridging oxygen bonding $\mathrm{Si}-\mathrm{O}-\mathrm{Pb}(\mathrm{NBO})$, and the oxygen bonding which is linking the two lead atoms $\mathrm{Pb}-\mathrm{O}-\mathrm{Pb}$ (denoted as $\mathrm{MBO}$ - metal bridging oxygen). The first type of bonding usually dominates in low-lead glasses, the third one is most characteristic for high-lead glasses. In the composition range of $x=0.50-0.55$ the NBO's prevail. It is clear, that the concentration dependences of these structural motifs are highly correlated with those obtained by us for the PLintensity dependencies of the PL-bands described above. On this basis, we assume that the luminescence centers are associated with these structural unit groups, namely the $R$-band is assigned to electron radiative transitions in the structural fragments with 
MBO's, the O-band - to that with NBO's and the B-band - to the BO's.

As shown earlier, the top valence band in low-lead glasses is formed by the $\mathrm{Pb} 6 s$ states $[7,8]$. High-levels of the valence band in high-lead glassy matrix are also formed by $6 s$ electron levels of lead, but because of the $\mathrm{Pb}-\mathrm{O}-\mathrm{Pb}$ and $\mathrm{Si}-\mathrm{O}-\mathrm{Pb}$ bonding the $02 p$ partial density of states make a significant donation to the valence band edge. In this case, the bottom of conduction band in the whole range of $x$ compositions is formed by $\mathrm{Pb} 6 p$ states. Since all three bands are excited in the fundamental absorption edge, it seems reasonable to assume that the PL-spectrum originates from the electron transitions with relevant localized tail-states: $\mathrm{Pb}$ $6 p \rightarrow \mathrm{Pb} 6 s$ (the $B$-band), $\mathrm{Pb} 6 p \rightarrow \mathrm{NBO} 2 p$ (the O-band) and $\mathrm{Pb}$ $6 p \rightarrow$ MBO $2 p$ (the $R$-band).

Some luminescence features, in particular thermal quenching, are essentially determined by tail-states, whose presence is a consequence of additional disordering in the glassy system. It is seen from Fig. 5 that the temperature quenching curves are the subject of the known Street law, which is characteristic for the continuum of disordered systems. The $T_{0}$ factor of Street function reflects the presence of dispersion in structural and energy parameters of the luminescence centers. As follows from Table. 1 , the $T_{0}$ parameter is different for different types of centers, and has smaller values in the case of $O$-band than in the $B$-band. This means that the energy parameters scattering of different centers is strongly linked with their nature. The difference in the disordering of the nearest environment is manifested usually in the existence of centers distribution on the activation energy. The specific of the obtained distributions is the presence of a significant part of centers with a low meaning of activation energy. This fact determines the dependence character when the process of luminescence quenching starts even from the lowest temperatures. The width of the energy distributions, presented in Table 2, clearly reflects the disorder in the immediate neighborhood centers, but not the matrix as a whole.

The features of the excitation and radiative relaxation of the involved three types of localized states are shown schematically in Fig. 8. Even with the same energy of exciting photons, one can realize the different types of radiative transitions, giving a band of $R, O$ and $B$-type in the PL-spectra. In this case, the particular type of electron radiative transition will be determined by the composition of the matrix, the predominant type of structural fragment (BO, NBO or MBO), and, hence, the nature of localized states in the tails of the energy bands.

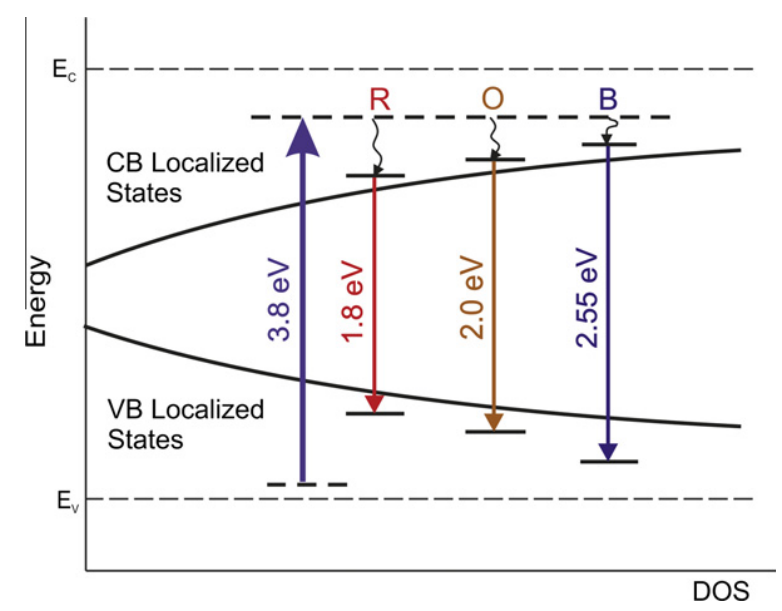

Fig. 8. Excitation and radiative relaxation scheme proposed for three types of localized states in $x \mathrm{PbO} \cdot(1-x) \mathrm{SiO}_{2}$ glasses. Wavy arrows denote a relaxation of photoexcited states in the tail of conduction band. $B$-band is $\mathrm{Pb} 6 p \rightarrow \mathrm{Pb} 6 s$ transition; 0 -band is $\mathrm{Pb} 6 p \rightarrow \mathrm{NBO} 2 p$ transition and $R$-band is $\mathrm{Pb} 6 p \rightarrow \mathrm{MBO} 2 p$ transition.

\section{Conclusion}

The results of our study demonstrate that the excitation of luminescence spectrum near the fundamental absorption edge of the binary lead silicate glasses is due to a superposition of three emission bands at $1.8 \mathrm{eV}, 2.0 \mathrm{eV}$ and $2.55 \mathrm{eV}$. Observed emission was interpreted as the radiative relaxation of exciton-like excitations in the tails of the allowed energy bands. The origin of these bands is associated with localized states of different structural fragments of the glassy network, each of which is determined by a certain type of oxygen atoms (MBO, NBO and $\mathrm{BO}$ ). The $2.55 \mathrm{eV}$ luminescence, that is the most characteristic for low-lead glasses, was attributed radiative transition $\mathrm{Pb} 6 p \rightarrow \mathrm{Pb} 6 s$ in lead ions. In the case of high-lead glasses the lead-ions become glass-forming units. The main contribution to the PL process is determined by the $\mathrm{Pb} 6 p \rightarrow$ MBO $2 p(1.8 \mathrm{eV})$ transitions between the tail-states of the conduction band and the oxygen levels in the structural fragments of $\mathrm{Pb}-\mathrm{O}-\mathrm{Pb}$ type. In the range of intermediate compositions the luminescence due to $\mathrm{Pb} 6 p \rightarrow$ NBO $2 p$ transitions is dominated.

Finally, it was established that the temperature dependence of the intrinsic luminescence in the studied glasses obeys the empirical Street law, the implementation of which is the characteristic of many disordered systems. The presentation of the temperature dependence as a superposition of discrete Street-Mott relationships allows to obtain the distribution of emission centers on the activation energy of quenching process. The shape of received distributions is asymmetrical and has a high contribution of low-energy states experiencing practically non-activated luminescence quenching at low temperatures. At the same time the different width of the energy distributions for a nonequivalent luminescence centers reflects their dissimilar atomic and energy as well as the degree of disorder in the near surroundings.

\section{Acknowledgments}

This study was supported by the Russian Foundation for Basic Research (Project No. 09-02-00493).The authors thank Dr. V.A. Pustovarov for the technical support in luminescence experiment.

\section{References}

[1] P. Roth, G.W. Fraser, Nuclear Instruments and Methods in Physics Research A 439 (2000) 134

[2] J.L. Wiza, Nuclear Instruments and Methods 162 (1979) 587.

[3] C.C. Wang, Physical Review B 2 (1970) 2045.

[4] O.M. Efimov, Yu A. Matveev, A.M. Mekryukov, Quantum Electronics 24 (1994) 311.

[5] A.F. Zatsepin, V.S. Kortov, V. Yu, Shchapova Radiotekhnika i Elektronika 37 (1992) 326

[6] A.N. Trukhin, Journal of Non-Crystalline Solids 189 (1995) 1.

[7] V.A. Gubanov, A.F. Zatsepin, V.S. Kortov, et al., Journal of Non-Crystalline Solids 127 (1991) 259.

[8] A.L. Shakhmin, A.M. Tyutikov, Fizika i Khimiya Stekla 16 (1990) 833.

[9] K.N. Dalby, H.W. Nesbitt, V.P. Zakaznova-Herzog, et al., Geochimica and Cosmochimica Acta 71 (2007) 4297.

[10] A.M. Tyutikov, N.B. Lobanova, M.N. Toiseva, et al., Fizika i Khimiya Stekla 5 (1979) 628.

[11] A.F. Zatsepin, A.I. Kukharenko, D.A. Zatsepin, et al., Optical Materials 33 (2011) 601.

[12] A.N. Trukhin, Journal of Non-Crystalline Solids 123 (1990) 250.

[13] L.B. Glebov, A.G. Plyukhin, E.L. Raaben, et al., Fizika i Khimiya Stekla 16 (1990) 245.

[14] C. Bettinali, G. Ferraresso, Journal of Non-Crystalline Solids 1 (1968) 91.

[15] C. Bettinali, V. Gottardi, B. Locardi, Journal of Non-Crystalline Solids 1 (1969) 360.

[16] R.A. Street, Advances in Physics 25 (1976) 397.

[17] R.A. Street, Physical Review B 21 (1980) 5775.

[18] A.F. Zatsepin, A.I. Kukharenko, E.A. Buntov, et al., Glass Physics and Chemistry 36 (2010) 166.

[19] A.F. Zatsepin, E.A. Buntov, A.L. Ageev, Journal of Luminescence 130 (2010) 1721. 